\section{La renovación de la atención primaria de salud en las Américas: la propuesta de la Organización Panamericana de la Salud para el siglo XXI}

Mirta Roses Periago'

1 Directora, Oficina Ssanitaria Panamericana, Washington, D.C., Estados Unidos de América.
El número especial de la Revista Panamericana de Salud Públical Pan American Journal of Public Health (RPSP/PAJPH) que tengo el gusto de presentar está dedicado a la atención primaria de salud (APS) y forma parte del proceso que lidera la Organización Panamericana de la Salud (OPS) para renovar la APS en la Región de las Américas.

La Conferencia Internacional sobre Atención Primaria de Salud, celebrada en 1978 en Alma-Ata (Kazajstán, Unión Soviética), definió y otorgó el reconocimiento internacional al concepto de APS como principal estrategia para alcanzar la meta de Salud Para Todos en el Año 2000. Esta visión sobre la APS quedó expresada en los principios y recomendaciones contenidos en la Declaración de Alma-Ata, que marcaron el inicio de un nuevo paradigma para mejorar la salud colectiva y el establecimiento de una nueva plataforma de política sanitaria internacional (1).

Desde 1978, el contexto general en la Región ha cambiado notablemente, no solo por los propios procesos internos de los países, sino también por las modificaciones ocurridas a escala mundial que inciden sobre los individuos y las poblaciones. Los cambios ocurridos desde los puntos de vista epidemiológico, demográfico, social, político y medioambiental son ostensibles y han generado diferentes escenarios sobre los cuales aparecen nuevos riesgos que amenazan a la salud y - junto a los viejos problemas todavía pendientes de resolver- exigen nuevas estrategias. Esta compleja situación reafirma la importancia que se le reconoció a la APS en 1978.

Las relaciones entre la salud y el desarrollo son indiscutibles y se reconoce que la salud es un resultante del proceso social, económico y político y que es también un derecho humano fundamental. A su vez la buena salud tiene un efecto benéfico sobre el nivel educacional, la productividad en el trabajo y el bienestar general de la población (2). En tanto, la desigualdad, la pobreza, la explotación, la violencia y la injusticia impactan negativamente en la salud (3). Debido a esta estrecha relación entre la salud y el desarrollo, los enfoques dirigidos a mejorar la salud deben tomar en cuenta necesariamente sus múltiples factores determinantes de una forma integrada y orientarse a corregir estos factores que, en muchos casos, trascienden el ámbito estrictamente sanitario. La APS es la estrategia idónea para actuar en este complejo proceso de generación y protección de la salud individual y colectiva, ya que aborda la situación de los individuos y las familias como un todo y toma en cuenta las circunstancias del entorno en el que se desenvuelven. El llamamiento de la APS para analizar de manera conjunta con otros sectores los determinantes de la salud y las enfermedades abre el camino hacia la formulación de políticas públicas conducentes al desarrollo humano integral y sostenible. 
Aunque en la Región, en general, se ha avanzado considerablemente en el mejoramiento de diversos indicadores de salud durante los últimos decenios, aún persisten desigualdades y grandes diferencias, tanto entre los países como entre los diversos grupos de la población de un mismo país (4). Los Objetivos de Desarrollo del Milenio, adoptados en 2000 por acuerdo de 189 países (5), constituyen un conjunto de objetivos y metas cuantificables dirigidos a combatir la pobreza, el hambre, las enfermedades, el analfabetismo, la degradación del medio ambiente y la discriminación contra la mujer. Los mecanismos necesarios para alcanzar esos objetivos y metas en 2015 se complementan y se refuerzan mutuamente con las estrategias de APS. A su vez, la APS es el entorno ideal para la operativización de las estrategias encaminadas a lograr los Objetivos de Desarrollo del Milenio, ya que se basan en los mismos principios y valores de equidad, solidaridad y justicia social, convoca a la participación social y reconoce el tremendo aporte de la ciencia y la tecnología con control social para su pleno aprovechamiento en beneficio del progreso humano.

"Las relaciones entre la salud y el desarrollo son
indiscutibles y se reconoce que la salud es un
resultante del proceso social, económico y
politico y que es también un derecho humano
fundamental."

La contribución de la APS al mejoramiento de la salud de la población - como consecuencia de un mayor acceso a los servicios, de la aplicación de un enfoque preventivo y de contar con personal más capacitado- ha sido ampliamente reconocida por la comunidad internacional (6). ${ }^{2}$ Precisamente, durante las últimas décadas se han "cosechado" en las Américas diversas experiencias sobre la expansión de la APS, con la adopción de medidas preventivas y mejoras en la educación, el saneamiento y el acceso a los servicios de salud. Todo ello ha permitido mejorar los indicadores de salud y de desarrollo social en la Región (7-11).

Al comienzo del nuevo siglo, la OPS ha convocado a reuniones y acuerdos internacionales para resaltar la necesidad de reorientar los sistemas y servicios ante los requerimientos planteados por el nuevo contexto regional. Entre ellas se pueden destacar el Taller Regional sobre la Atención Primaria de Salud, celebrado en Brasilia, Brasil, en 2001; la Declaración de Brasilia (5), de 2003; la reunión del Grupo de Trabajo de Atención Primaria de Salud, celebrada en Washington, D.C., Estados Unidos de América, en 2003; la Declaración de Boca Chica, República

\footnotetext{
2 McGuire J. Social provisioning, socioeconomic context, and child mortality: a cross-national analysis. Annual Meeting of the American Political Science Association; 2002 Aug 29-Sep 1; Boston, Massachusetts [documento no publicado].
} 
Dominicana, de 2004 (12); y la reunión del Grupo de Trabajo de Atención Primaria de Salud, celebrada en San José, Costa Rica, en 2004. En 2003, coincidiendo con la celebración del $25 .^{\circ}$ aniversario de la Conferencia de Alma-Ata, se inició un proyecto dirigido a fortalecer la APS en los Estados Miembros de la OPS.

“... la atención primaria de salud es el entorno ideal para la operativización de las estrategias encaminadas a lograr los Objetivos de Desarrollo del Milenio, ya que se basan en los mismos principios y valores de equidad, solidaridad y justicia social ..."

De acuerdo con la Resolución CD44.R6, aprobada por el $44 .^{\circ}$ Consejo Directivo de la OPS en septiembre de 2003, se creó en mayo de 2004 el Grupo de Trabajo de Atención Primaria de Salud, con el objetivo de promover la reorientación de los sistemas y servicios de salud en la Región de acuerdo con los principios de Alma-Ata. El grupo elaboró un importante documento de posición basado en las pruebas científicas resultantes de la revisión sistemática de la literatura y del análisis exhaustivo realizado por expertos de dentro y fuera de la Región (13). Durante la Reunión Regional de Consulta sobre la Renovación de la Atención Primaria de Salud en las Américas, celebrada en Montevideo, Uruguay, en julio de 2005, se preparó una Declaración Regional sobre las nuevas orientaciones de la atención primaria de salud, que fue ratificada por el $46 .^{\circ}$ Consejo Directivo de la OPS en septiembre de ese mismo año.

"La propuesta de la OPS implica renovar
integralmente los sistemas de salud, tomando la
atención primaria de salud como fuente principal
de atención y cuidados sanitarios."

Actualmente, la APS ha recuperado visibilidad en todo el mundo: las instituciones gubernamentales, las entidades públicas y privadas, las organizaciones de cooperación internacional y del ámbito académico, entre otras, reconocen que el fortalecimiento de los sistemas sanitarios es un requisito imprescindible para asegurar el crecimiento económico, avanzar en materia de equidad social y mejorar la salud.

La propuesta de la OPS implica renovar integralmente los sistemas de salud, tomando la APS como fuente principal de atención y cuidados sanitarios. Esta transformación se debe diseñar según las necesidades de la población y las características específicas de cada país y se debe dotar a los sistemas y servicios de salud de la tecnología apropiada para responder a la mayoría de los problemas de salud de la comunidad. 
Este número monográfico de la RPSP/PAJPH cuenta con dos partes bien diferenciadas, pero complementarias entre sí. En primer lugar se expone la perspectiva de la OPS sobre el papel que debe desempeñar la APS como estrategia para mejorar la equidad y el desarrollo, expresada en el documento de posición y en la Declaración Regional sobre la Atención Primaria de Salud. La segunda parte del volumen contiene una serie de artículos que presentan el panorama relacionado con el desarrollo y funcionamiento de la APS en la Región de las Américas. Ambas partes contribuirán, sin duda, a dar al lector una visión más amplia de las distintas facetas de la APS.

El legado político y ético de Alma-Ata, las lecciones aprendidas y las buenas prácticas acumuladas a lo largo de treinta años nos serán de gran utilidad para afrontar con éxito los desafíos del siglo XXI.

\section{REFERENCIAS}

1. Declaración de Alma-Ata. En: Alma-Ata 1978: atención primaria de salud. Informe de la Conferencia Internacional sobre Atención Primaria de Salud; septiembre 6-12 de 1978; Alma-Ata, URSS. Ginebra: Organización Mundial de la Salud; 1978. Pp. 3-4. Hallado en: http://www.paho.org/spanish/dd/pin/Alma Ata_declaracion.htm. Acceso el 31 de julio de 2006.

2. World Health Organization. Macroeconomics and health: investing in health for economic development. Geneva: WHO; 2001.

3. People's Health Movement, ed. Health for all now! Revive Alma Ata!! The Alma Ata anniversary pack. Bangalore: People's Health Movement; 2003.

4. Schneider MC, Castillo-Salgado C, Loyola-Elizondo E, Bacallao J, Mujica OJ, Vidaurre M, et al. Trends in infant mortality inequalities in the Americas: 1955-1995. J Epidemiol Community Health. 2002;56(7):538-41.

5. Organización Panamericana de la Salud. Atención primaria de salud. Los Objetivos de Desarrollo del Milenio en las Américas. Bol Epidemiol. 2004;25(2). Hallado en: http:/ / www.paho.org/spanish/dd/ais/be_v25n2-mdgs.htm. Acceso el 31 de julio de 2006.

6. Caminal J, Starfield B, Sánchez E, Casanova C, Morales M. The role of primary care in preventing ambulatory care sensitive conditions. Eur J Public Health. 2004;14(3):246-51.

7. Klijzing F, Taylor H. The decline in infant mortality in Costa Rica, 1950-1973: modernization or technological diffusion? Malays J Trop Geogr. 1982;5:22-9.

8. Rosero-Bixby L. Infant mortality in Costa Rica: explaining the recent decline. Stud Fam Plann. 1986;17:57-65.

9. Spiegel JM, Yassi A. Lessons from the margins of globalization: appreciating the Cuban health paradox. J Public Health Policy. 2004;25(1):85-110.

10. Castañeda T. Determinantes del descenso de la mortalidad infantil en Chile, 19751982. Cuad Econ. 1985;22:195-214.

11. Svitone E, Garfield R, Vasconcelos M, Craveiro V. Primary health care lessons from the Northeast of Brazil. Rev Panam Salud Publica. 2000;7(5):293-302.

12. Organización Panamericana de la Salud. Declaración de Boca Chica. Reunión del Sector Salud de Centroamérica y República Dominicana; 8 de julio de 2004; Boca Chica, República Dominicana. Hallado en: http://www.cor.ops-oms.org/ TextoCompleto/documentos/Declaración de Boca Chica.pdf. Acceso el 31 de julio de 2006.

13. Pan American Health Organization. Primary health care remains key. Hallado en: http://www.paho.org/English/DD/PIN/ptoday12_nov04.htm. Acceso el 31 de julio de 2006. 